\title{
Interhemispheric transchoroidal approach to resect third ventricular teratoma
}

\author{
Yuki Ito, MD, ${ }^{1}$ Tomohiro Inoue, MD, PhD, ${ }^{1}$ Akira Tamura, MD, PhD, ${ }^{1}$ \\ and Kazuo Tsutsumi, MD, PhD² \\ 'Department of Neurosurgery, Fuji Brain Institute and Hospital, Shizuoka, Japan; and \\ 2Department of Neurosurgery, Showa General Hospital, Tokyo, Japan
}

The authors demonstrate an interhemispheric transchoroidal approach for third ventricular teratoma resection. Interhemispheric dissection exposed the corpus callosum at a length of about $2 \mathrm{~cm}$. A callosotomy was made to enter into the right lateral ventricle. After septal vein ligation, dissection was made of the space between the right fornix and right internal cerebral vein (ICV); thus bilateral fornix and left ICV would be retracted to the left; right choroid plexus, right ICV to the right. By this transchoroidal approach, the foramen of Monro was extended posteriorly, providing enough of a surgical corridor to resect a posteriorly located third ventricular tumor.

The video can be found here: https://youtu.be/glzPiH3zx_o.

KEYWORDS transchoroidal; third ventricle; interhemispheric; video 